\title{
Cycling of gut mucosal CD4 + T cells decreases after prolonged anti-retroviral therapy and is associated with plasma LPS levels
}

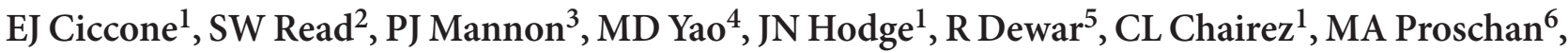 \\ JA Kovacs ${ }^{7}$ and I Sereti ${ }^{1}$
}

The gut mucosa is an important site of HIV immunopathogenesis with severe depletion of CD4 + T cells occurring during acute infection. The effect of prolonged anti-retroviral therapy (ART) on cycling and restoration of T lymphocytes in the gut remains unclear. Colon and terminal ileal biopsies and peripheral blood samples were collected from viremic, untreated, HIV-infected participants, patients treated with prolonged ART ( $>5$ years), and uninfected controls and analyzed by flow cytometry. In the gut, the proportion of cycling T cells decreased and the number of CD4 + T cells normalized in treated patients in parallel with $\beta 7$ expression on CD4 + T cells in blood. Cycling of gut T cells in viremic patients was associated with increased plasma LPS levels, but not colonic HIV-RNA. These data suggest that gut T-cell activation and microbial translocation may be interconnected whereas prolonged ART may decrease activation and restore gut CD4 + T cells.

\section{INTRODUCTION}

The gut mucosa is an important site of early SIV and HIV pathogenesis. The majority of CD $4+\mathrm{T}$ cells in the gut express CCR5, the co-receptor for HIV, and show an activated, memory phenotype presumably because of constant exposure to dietary and bacterial antigens. ${ }^{1-3}$ HIV preferentially infects activated, memory CD4 + T cells; therefore, gut mucosal T lymphocytes provide a large pool of target cells that are susceptible to HIV infection and supportive of active HIV replication. ${ }^{1-7}$ During acute SIV and HIV infection, gut mucosal CD $4+\mathrm{T}$ cells are rapidly and preferentially depleted, primarily in the lamina propria (LP), before $\mathrm{HIV}$-mediated changes are observed in peripheral blood or other lymphoid tissues such as lymph nodes and the spleen. ${ }^{4,6,8-14}$ Gut $\mathrm{CD} 4+\mathrm{T}$-cell depletion and direct HIV effects on the gut epithelium $^{15}$ can disrupt its integrity allowing for the translocation of microbial products, a phenomenon that has been associated with immune activation in chronically infected patients. ${ }^{16}$
The degree to which the CD4 + T-cell population in the gut can be reconstituted with anti-retroviral therapy is still unclear. There is evidence indicating that in response to less than 4 years of anti-retroviral therapy (ART), CD $4+$ T-cell restoration in the gut is delayed and incomplete, regardless of the stage of infection during which ART is initiated. ${ }^{4,10,17,18}$ However, recent studies, some evaluating the effects of long-term ( $>4$ years) ART, have suggested that restoration of gut mucosal CD $4+\mathrm{T}$ cells can occur ${ }^{19,20}$ and may happen more rapidly if treatment is initiated during acute infection. ${ }^{21-23}$ Importantly, many of these studies have quantified immune reconstitution in response to ART only by relative proportions of T cells. This may result in an underestimation of CD4 + T-cell recovery because of significant expansion or accumulation of CD8 + T cells. ${ }^{24,25}$ In addition, evidence from the SIV model has suggested that gut CD4 + T-cell loss may be reflected in the proportion of peripheral blood lymphocytes expressing high levels of the lymphocyte homing

\footnotetext{
${ }^{1}$ Laboratory of Immunoregulation, National Institute of Allergy and Infectious Diseases, Bethesda, Maryland, USA. ${ }^{2}$ The Division of AIDS, National Institute of Allergy and Infectious Diseases, Bethesda, Maryland, USA. ${ }^{3}$ Division of Gastroenterology and Hepatology, University of Alabama-Birmingham, Birmingham, Alabama, USA. ${ }^{4}$ Laboratory of Host Defenses, National Institute of Allergy and Infectious Diseases, Bethesda, Maryland, USA. ${ }^{5}$ SAIC-Frederick Inc., Frederick, Maryland, USA. ${ }^{6}$ The Biostatistics Research Branch, National Institute of Allergy and Infectious Diseases, Bethesda, Maryland, USA. ${ }^{7}$ The Critical Care Medicine Department, NIH, Bethesda, Maryland, USA. Correspondence: I Sereti (isereti@niaid.nih.gov) 
marker, $\alpha 4 \beta 7 ;^{26}$ this association has yet to be evaluated in human HIV infection in the absence or presence of ART.

Immunophenotype and gene expression profiles of gut mucosa T lymphocytes indicate that both the CD $4+$ and CD8 + T-cell activation observed in HIV infection persist in the gut despite ART. ${ }^{17,21}$ However, there are limited data characterizing the effect of therapy on T-cell cycling and the relationship between $\mathrm{T}$-cell proliferation and reconstitution. It is known that HIV infection causes increased cycling of CD $4+$ and CD8 + T cells in peripheral blood and lymph nodes that decreases in response to ART. ${ }^{27-29}$ In the gut mucosa, immunohistochemical analysis has suggested that HIV causes an increase in T-cell proliferation that persists despite short-term treatment, but may normalize with prolonged (3-7 years) therapy. ${ }^{17}$

The primary goal of this study was to measure immune restoration and T-cell cycling in the colon and terminal ileum (TI) of HIV-infected patients and to evaluate the potential stimuli of T-cell cycling, both in the peripheral blood and at the tissue level in the gut mucosa. In addition, we evaluated potential associations between the proportion of CD $4+\mathrm{T}$ cells expressing $\beta 7$ integrin in peripheral blood and gut CD $4+\mathrm{T}$-cell populations. We observed that significant CD4 + T-cell restoration can occur in the gut (that parallels $\beta 7$ expression in blood) after prolonged ART. In addition, the proportion of cycling CD4 $+\mathrm{T}$ cells decreases or normalizes in the gut after prolonged ART and is associated with plasma lipopolysaccharide (LPS) levels suggesting that gut immune activation and microbial translocation may be interconnected at the tissue level.

Table 1 Clinical characteristics of study participants

\begin{tabular}{|c|c|c|c|}
\hline & HIV-(n=10) & No $\operatorname{ART}(n=14)$ & $\mathrm{VL}<50(n=12)$ \\
\hline $\begin{array}{l}\text { Age } \\
\text { CD4 + T cells } \\
\text { per } \mu l\end{array}$ & $\begin{array}{c}46(40-50) \\
773 \\
(496-1,359)\end{array}$ & $\begin{array}{c}47(35-49) \\
175 \\
(37-263)\end{array}$ & $\begin{array}{c}48(41-50) \\
535 \\
(384-608)\end{array}$ \\
\hline $\begin{array}{l}\% \text { CD4 }+\mathrm{T} \\
\text { cells }\end{array}$ & $\begin{array}{c}45 \\
(38-49)\end{array}$ & $\begin{array}{c}12 \\
(6-26)\end{array}$ & $\begin{array}{c}32 \\
(23-38)\end{array}$ \\
\hline $\begin{array}{l}\text { CD8 }+ \text { T cells } \\
\text { per } \mu l\end{array}$ & $\begin{array}{c}399 \\
(313-443)\end{array}$ & $\begin{array}{c}755 \\
(334-919)\end{array}$ & $\begin{array}{c}703 \\
(520-988)\end{array}$ \\
\hline$\%$ CD8 + T cells & $\begin{array}{c}20 \\
(19-25)\end{array}$ & $\begin{array}{c}60 \\
(53-68)\end{array}$ & $\begin{array}{c}42 \\
(36-53)\end{array}$ \\
\hline CD4:CD8 ratio & $\begin{array}{c}2.13 \\
(1.59-2.58)\end{array}$ & $\begin{array}{c}0.19 \\
(0.07-0.50)\end{array}$ & $\begin{array}{c}0.72 \\
(0.45-1.04)\end{array}$ \\
\hline $\begin{array}{l}\text { Plasma HIV- } \\
\text { RNA (copies } \\
\text { per ml) }\end{array}$ & $N / A^{b}$ & $\begin{array}{c}31,199 \\
(6,505- \\
216,531)\end{array}$ & $<50$ \\
\hline $\begin{array}{l}\text { Colon tissue } \\
\text { HIV-RNA } \\
\text { (copies per mg) }\end{array}$ & $\mathrm{N} / \mathrm{A}$ & $\begin{array}{c}180^{\mathrm{b}} \\
(49-2,645)\end{array}$ & $<50$ \\
\hline Years on ART & $\mathrm{N} / \mathrm{A}$ & $N / A$ & $\begin{array}{c}8 \\
(5-10)\end{array}$ \\
\hline $\begin{array}{l}\text { Nadir CD4 }+\mathrm{T} \\
\text { cells per } \mu \mathrm{l}\end{array}$ & N/A & N/A & $\begin{array}{c}187 \\
(36-270)\end{array}$ \\
\hline
\end{tabular}

aTissue HIV-RNA only available for 11 of the No ART participants.

bParticipants with HIV-RNA levels below the limit of detection were assigned a

value of 49 copies per milligram.

Median values with IQR in parentheses.

N/A: Not applicable.

\section{RESULTS}

\section{Clinical characteristics of patient groups}

Clinical characteristics of the three groups are shown in Table $\mathbf{1}$. Nine of the viremic patients were ART-naive, whereas five had a therapy interruption of at least 6 months duration before biopsy. Subgroup analysis of ART-naive patients of the No ART group yielded similar conclusions for the main statistical comparisons. All treated participants initiated ART during chronic infection and had been on therapy for a median of 8 years with virological suppression to $<50$ copies per milliliter for at least 2 years at the time of the biopsy. Reportable histopathological findings were observed in four No ART patients (one case of tubular adenoma, one case of focal acute colitis, and two cases of spirochetosis), four $\mathrm{VL}<50$ patients (two cases of tubular adenoma and two cases of focal acute colitis), and three HIV-uninfected controls (two cases of tubular adenoma and one case of spirochetosis). All participants were clinically stable and asymptomatic at the time of biopsy and did not require therapy for any of the above findings. Excluding patients with incidental findings on histopathological specimens (focal enteritis or spirochetosis) did not alter any of our main reported statistical analyses.

\section{The proportion of gut CD4 + T cells in treated patients was} lower than that of uninfected controls despite prolonged ART At both gastrointestinal (GI) sites, the proportion of CD4 + $\mathrm{T}$ cells in the No ART group was significantly lower than that of $\mathrm{VL}<50$ patients (colon: 5.9 vs. $38.3 \%$, TI: 6.6 vs. $30.3 \%$; $P<0.001$ for both comparisons) and controls (colon: 5.9 vs. $54.2 \%$, TI: 6.6 vs. 37.6\%; $P<0.001$ ) (Figure 1a). A similar pattern was observed in peripheral blood (Figure 1a). In addition, the percentage of $\mathrm{CD} 4+\mathrm{T}$ cells in the gut was lower in $\mathrm{VL}<50$ patients as compared with controls (colon: 38.3 vs. $54.2 \%, P=0.004$; TI: 30.3 vs. $37.6 \%, P=0.023$; Figure 1a).

The proportion of CD $8+\mathrm{T}$ cells was higher in viremic patients as compared with controls in both colon and TI (colon: 67.7 vs. $22.0 \%$, TI: 74.9 vs. $40.7 \%$; $P<0.001$ ) (Figure 1b). This was also true in comparison with the $\mathrm{VL}<50$ group (colon: 67.7 vs. $42.3 \%, P<0.001$; TI: 74.9 vs. $52.8 \%, P=0.015$; Figure 1b). As with CD $4+\mathrm{T}$ cells, the differences between groups were similar to those seen in peripheral blood (Figure 1b). The proportion of $\mathrm{CD} 8+\mathrm{T}$ cells of the $\mathrm{VL}<50$ group was higher than that of controls in the colon ( 42.3 vs. $22.0 \%, P=0.001$ ), but not in the TI.

\section{The absolute number of gut mucosal CD4 + T cells did not differ between uninfected controls and patients treated with prolonged ART}

In contrast to what was observed when comparing proportions of CD $4+\mathrm{T}$ cells, there was no statistically significant difference in the absolute number of $\mathrm{CD} 4+\mathrm{T}$ cells per gram of tissue between controls and the $\mathrm{VL}<50$ group in either colon or TI (colon: $3.6 \times 10^{6}$ vs. $3.9 \times 10^{6}, P=0.474$; TI: $1.6 \times 10^{6}$ vs. $1.0 \times 10^{6}$, $P=0.114$ ), mimicking the pattern seen in peripheral blood (Figure 1a). Fewer CD4 + T cells were observed in both colon and TI of viremic patients as compared with HIV-uninfected controls (colon: $0.56 \times 10^{6}$ vs. $3.6 \times 10^{6}, P=0.039$; TI: $0.29 \times 10^{6}$ vs. $\left.1.6 \times 10^{6} ; P=0.007\right)$ (Figure 1a). 
a
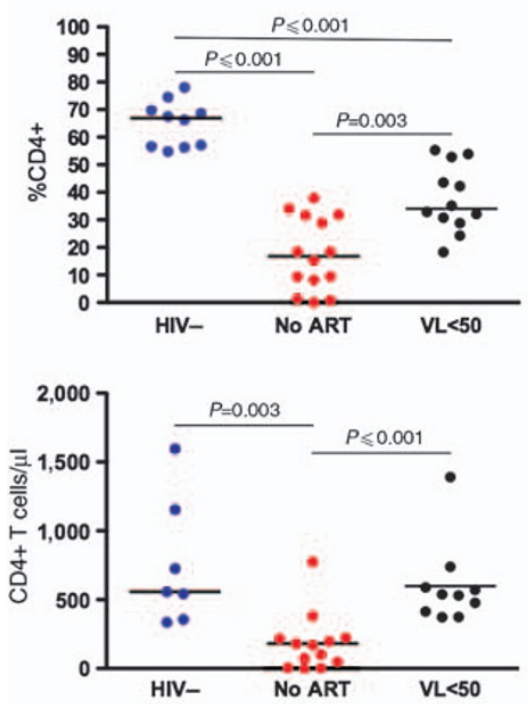

b
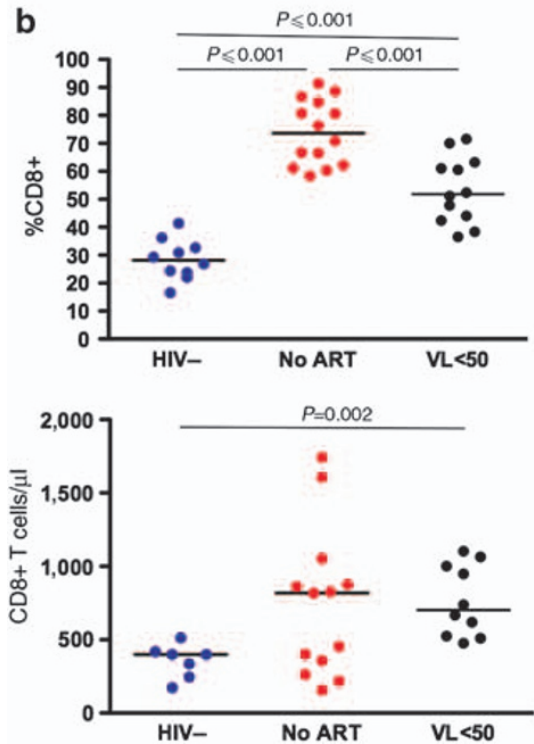

$\underline{\text { Colon }}$
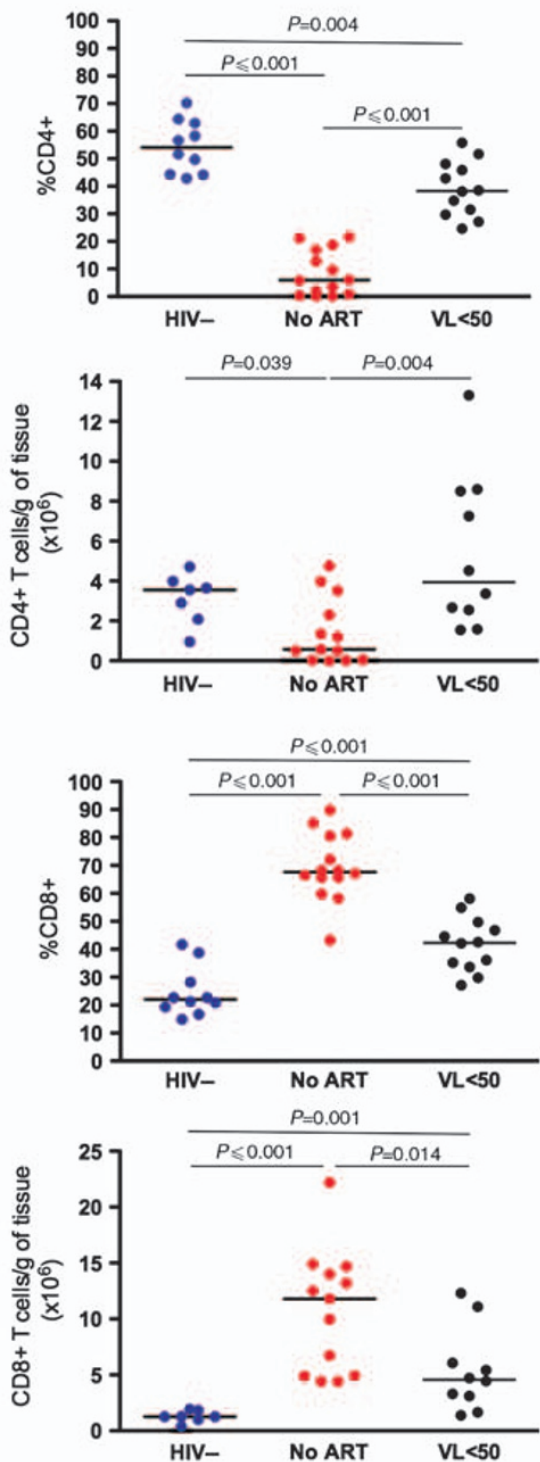

lleum
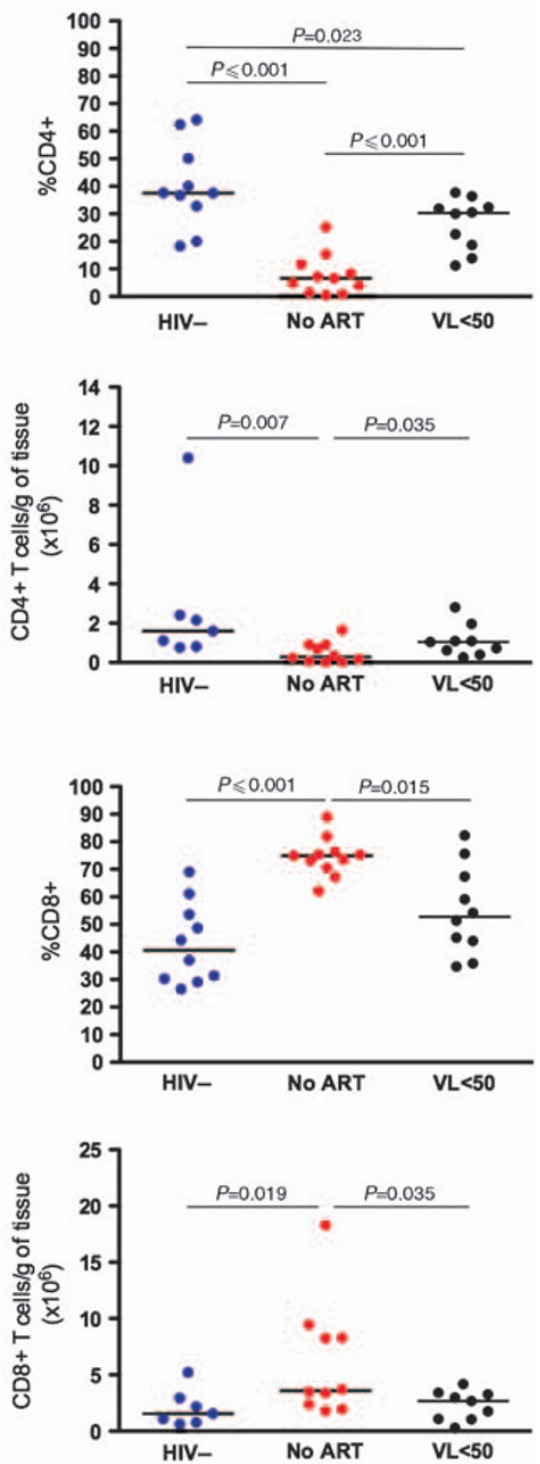

Figure 1 The proportion and absolute number of (a) CD4 + and (b) CD8 + T cells in peripheral blood and gut compartments. Percentages are proportions of $\mathrm{CD} 3+\mathrm{T}$ lymphocytes. Absolute numbers from gut are the number of cells per gram of biopsy tissue. Black bars indicate median values. Ileum values for the T-cell percentages were not available for one $\mathrm{VL}<50$ participant. Absolute number of estimates were available for $7 \mathrm{HIV}-$, $13 \mathrm{No}$ ART (10 for ileum), and $10 \mathrm{VL}<50$ (nine for ileum) patients.

At both gut sites, the absolute number of CD8 + T cells in the No ART group was higher than in the HIV-uninfected controls and $\mathrm{VL}<50$ patients, although the differences were more pronounced in the colon (colon, No ART: $11.8 \times 10^{6} \mathrm{vs}$. HIV-: $1.3 \times 10^{6}, P<0.001$ and vs. VL $<50: 4.6 \times 10^{6}, P=0.014$; TI, No ART: $3.6 \times 10^{6}$ vs. HIV-: $1.6 \times 10^{6}, P=0.019$ and vs. VL $<50$ : $\left.2.7 \times 10^{6}, P=0.035\right)$ (Figure 1b).

\section{The proportion and absolute number of CD4 + and CD8 +} $T$ cells differed between colon and terminal ileum

In controls and treated patients, the proportion of CD4 $+\mathrm{T}$ cells was significantly higher in colon compared with TI (HIV-, colon: 54.1 vs. TI: $37.6 \%, P=0.010$; $\mathrm{VL}<50$, colon: 38.3 vs. TI: $30.3 \%, P=0.006$; Supplementary Figure 1a). Conversely, the proportion of CD8 $+\mathrm{T}$ cells was significantly higher in the ileum. (HIV-, colon: $22 \%$ vs. TI: $40.7 \%, P=0.002$; VL $<50$, colon: $43.5 \%$ vs. TI: $52.8 \%, P=0.037$ ) (Supplementary Figure $1 \mathbf{b}$ ). In both of the HIV-infected groups, the absolute number of $\mathrm{CD} 4+$ (Supplementary Figure 1c) and CD8 + (Supplementary Figure 1d) T cells per gram of tissue was higher in colon as compared with ileum (CD4+ T cells, No ART, colon: $1.3 \times 10^{6}$ vs. TI: $0.29 \times 10^{6}, P=0.010$; VL $<50$, colon: $3.4 \times 10^{6}$ vs. TI: $1.0 \times 10^{6}$, $P=0.004$; CD8 + T cells, No ART, colon: $13 \times 10^{6}$ vs. TI: $3.6 \times 10^{6}$, $P=0.020$; VL $<50$, colon: $4.7 \times 10^{6}$ vs. TI: $\left.2.7 \times 10^{6}, P=0.008\right)$. The proportions of CD4 + (Supplementary Figure 1e) and CD8 + (Supplementary Figure 1f) T cells in colon and TI were strongly correlated across all three groups (\%CD4+: $r=0.86, P<0.001$; $\% \mathrm{CD} 8+: r=0.75, P<0.001)$. 
In peripheral blood, $\beta 7$ expression was higher on central memory as compared with effector memory CD4 + T cells, and was correlated with both the proportion and absolute number of CD4 + T cells in peripheral blood and both intestinal sites

The heterodimeric integrin, $\alpha 4 \beta 7$, is a homing receptor that facilitates migration of lymphocytes to the gut lamina propria and has been shown to bind to and signal through gp120 during HIV-1 infection. ${ }^{30,31}$ Previous research has shown that the majority of cells expressing high levels of $\beta 7$ also express $\alpha 4 .^{26}$ Therefore, the expression of $\beta 7$ integrin, as well as CCR5, the co-receptor for HIV, was quantified on peripheral blood Tlym- phocytes (gating strategy shown in Supplementary Figure 2). The proportion of T lymphocytes that were CD $4+\beta 7$ hi was lower in the No ART group than in either controls (0.62 vs. $4.2 \%, P<0.001)$ or treated individuals ( 0.62 vs. $2.0 \%, P=0.003)$; in addition, $\beta 7$ hi expression was lower in the $\mathrm{VL}<50$ group as compared with controls (2.0 vs. $4.2 \%, P=0.019$ ) (Figure 2a). A similar pattern was observed for CD4 + CCR5 + T cells, although there was no statistically significant difference between the HIV- and $\mathrm{VL}<50$ groups (Figure $2 \mathbf{b}$ ). The expression of these two surface markers was also measured on central and effector memory subsets (Figure 2c). In all three groups, $\beta 7$ hi expression (Figure 2d) was lower and expression of CCR5 (Figure 2e) was
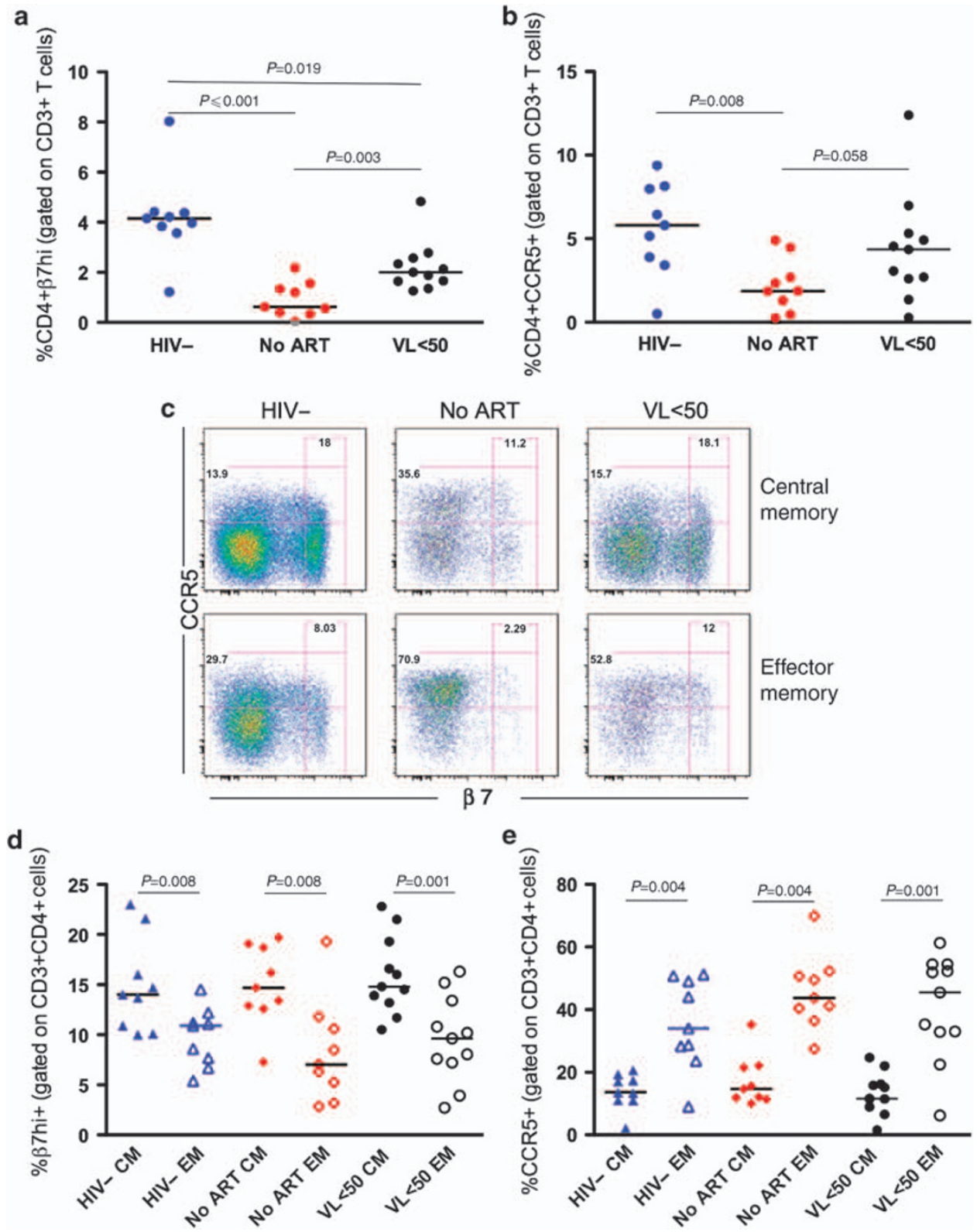

Figure 2 The proportion of peripheral blood CD4+ T cells expressing (a) $\beta 7$ hi and (b) CCR5 in 9 HIV-, 9 No ART, and 11 VL $<50$ participants. The gating strategy in part (c) was used to quantify the expression of (d) $\beta 7$ hi and (e) CCR5 on effector $(C D 27-C D 45 R O+)$ and central $(\mathrm{CD} 27+\mathrm{CD} 45 \mathrm{RO}+)$ memory $\mathrm{CD} 4+\mathrm{T}$ cells. Black bars indicate median values. 


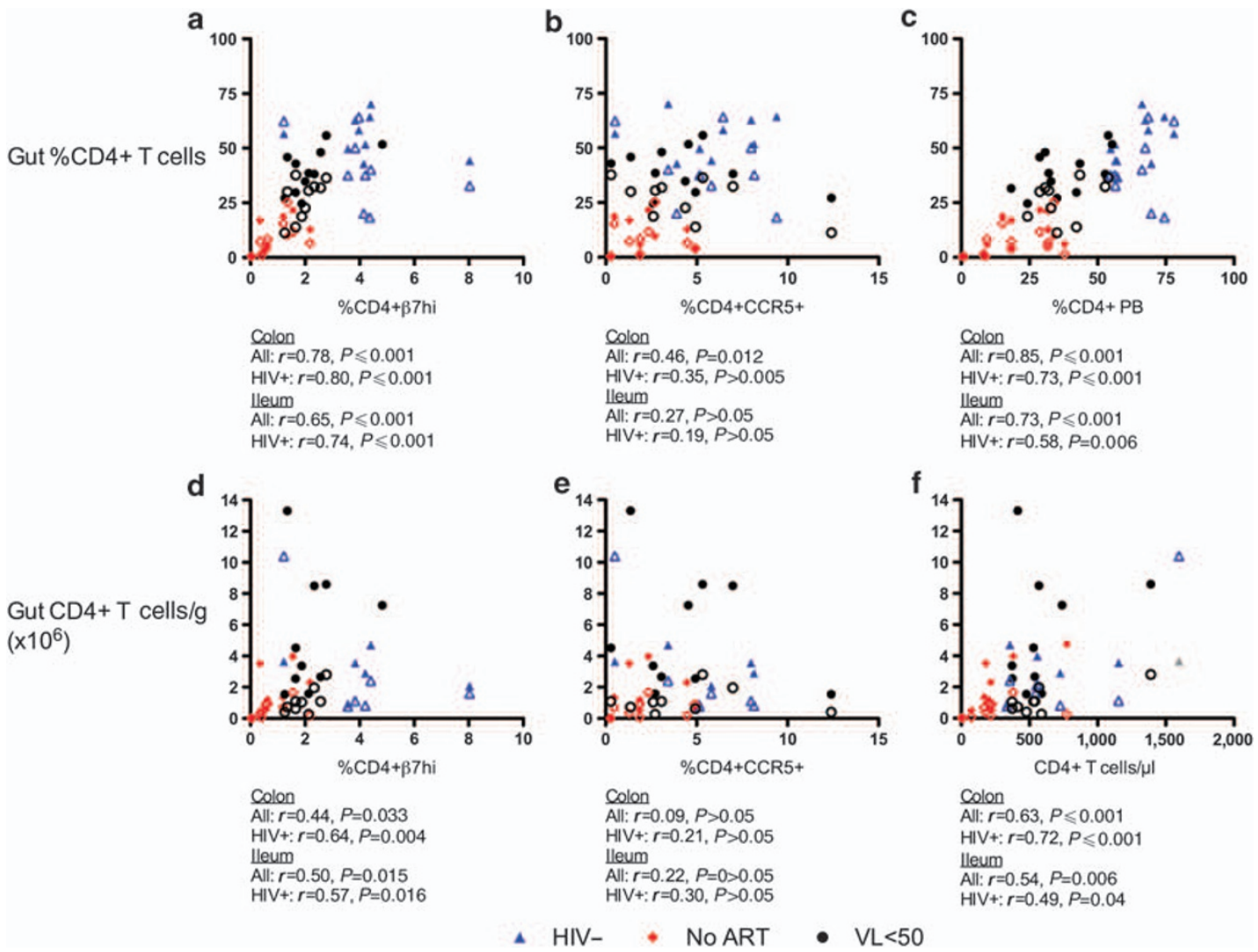

Figure 3 Comparison of the associations between peripheral blood (a, d) CD4+ $\beta 7 \mathrm{hi},(\mathbf{b}, \mathbf{e}) \mathrm{CD} 4+\mathrm{CCR} 5+$, and $(\mathbf{c}, \mathbf{f}) \mathrm{CD} 4+\mathrm{T}$ cells and the (a-c) percentage and (d-f) absolute number of CD4 + T cells in colon and terminal ileum. Filled symbols represent colon values, and open symbols reflect terminal ileal values. Spearman rank correlations for comparisons of all three groups and HIV + groups only are displayed below each graph.

higher in effector memory as compared with central memory CD4 + T-cell populations. There were no differences across study groups in either $\beta 7 \mathrm{hi}$ or CCR5 expression on central or effector memory CD $4+\mathrm{T}$ cells.

The proportion of $\beta 7$ hi CD $4+\mathrm{T}$ cells in peripheral blood correlated with both the percent and absolute number of CD4+ $\mathrm{T}$ cells in the periphery (data not shown) and at both gut sites (Figure 3a, d). The strongest correlations were observed when the analysis was restricted to HIV + groups only. In contrast, among HIV + individuals, the proportion of CCR $5+\mathrm{CD} 4+\mathrm{T}$ cells did not correlate with the percentage or absolute number of CD4 + T cells in the colon or TI (Figure $3 \mathbf{b}, \mathbf{e}$ ). However, the proportion and absolute number of CD4 + T cells in the PB did significantly correlate with the percent and absolute number of gut $\mathrm{CD} 4+\mathrm{T}$ cells, respectively (Figure $3 \mathbf{c}, \mathbf{f}$ ).

\section{In colon, the proportion of cycling CD4 + T cells was substantially decreased in response to prolonged ART and was not associated with colonic tissue HIV-RNA}

In both colon and ileum, the percentage of CD $4+\mathrm{T}$ cells that expressed Ki67 was significantly higher in untreated patients as compared with HIV-uninfected controls (colon: 12.0 vs. $2.2 \%$, TI: 12.0 vs. $1.9 \% ; P \leqslant 0.001$ ) and ART-treated patients (colon 12.0 vs. $3.0 \%$, TI: 12.0 vs. $3.9 \%$; $P \leqslant 0.001$ ) (Figure $4 a$ ). A similar pattern was observed in peripheral blood (Figure 4a). In colon and peripheral blood, there was no significant difference in the proportion of cycling CD4 $+\mathrm{T}$ cells between uninfected controls and treated patients (PB: 2.0 vs. $2.8 \%, P=0.110$; colon: 2.2 vs. $3.0 \%, P=0.055)$, however, the difference remained significant in TI (HIV-: $1.9 \%$ vs. $\mathrm{VL}<50: 3.9 \%$; $P=0.007$ ). The proportion of cycling CD8 + T cells did not differ between uninfected controls and treated patients in either peripheral blood or TI, but was slightly higher in controls (HIV-: $3.3 \%$ vs. $\mathrm{VL}<50: 2.6 \%$, $P=0.027$ ) in the colon (Figure $\mathbf{4 b}$ ). There were no statistically significant differences in the proportions of cycling CD4 + and CD8 + T cells between colon and ileum in any of the three study groups (Figure 4a, b).

To evaluate the relationship between T-cell cycling and immune restoration, we examined associations between the proportion of cycling $\mathrm{T}$ cells and both the proportion and absolute number of total CD4 + and CD8 + T cells in the HIVinfected groups. There was a strong inverse correlation between the percentage of $\mathrm{CD} 4+\mathrm{Ki} 67+\mathrm{T}$ cells and both the percentage of CD4 + T cells and CD4 + T-cell counts in PB (Table 2). In the colon, the percentage of CD4 + Ki67 + T cells inversely correlated with the proportion of $\mathrm{CD} 4+\mathrm{T}$ cells, but not with the number of $\mathrm{CD} 4+\mathrm{T}$ cells per gram of tissue. In $\mathrm{PB}$, the percentage of $\mathrm{CD} 8+\mathrm{Ki} 67+\mathrm{T}$ cells correlated with the proportion of CD8 + T cells, but not CD8 + T-cell count, and neither correlation was significant in the colon or TI (Table 2). Finally, $\beta 7$ hi expression on CD4 + T cells in PB inversely correlated with Ki67 expression on $\mathrm{CD} 4+\mathrm{T}$ cells in the peripheral blood, colon 

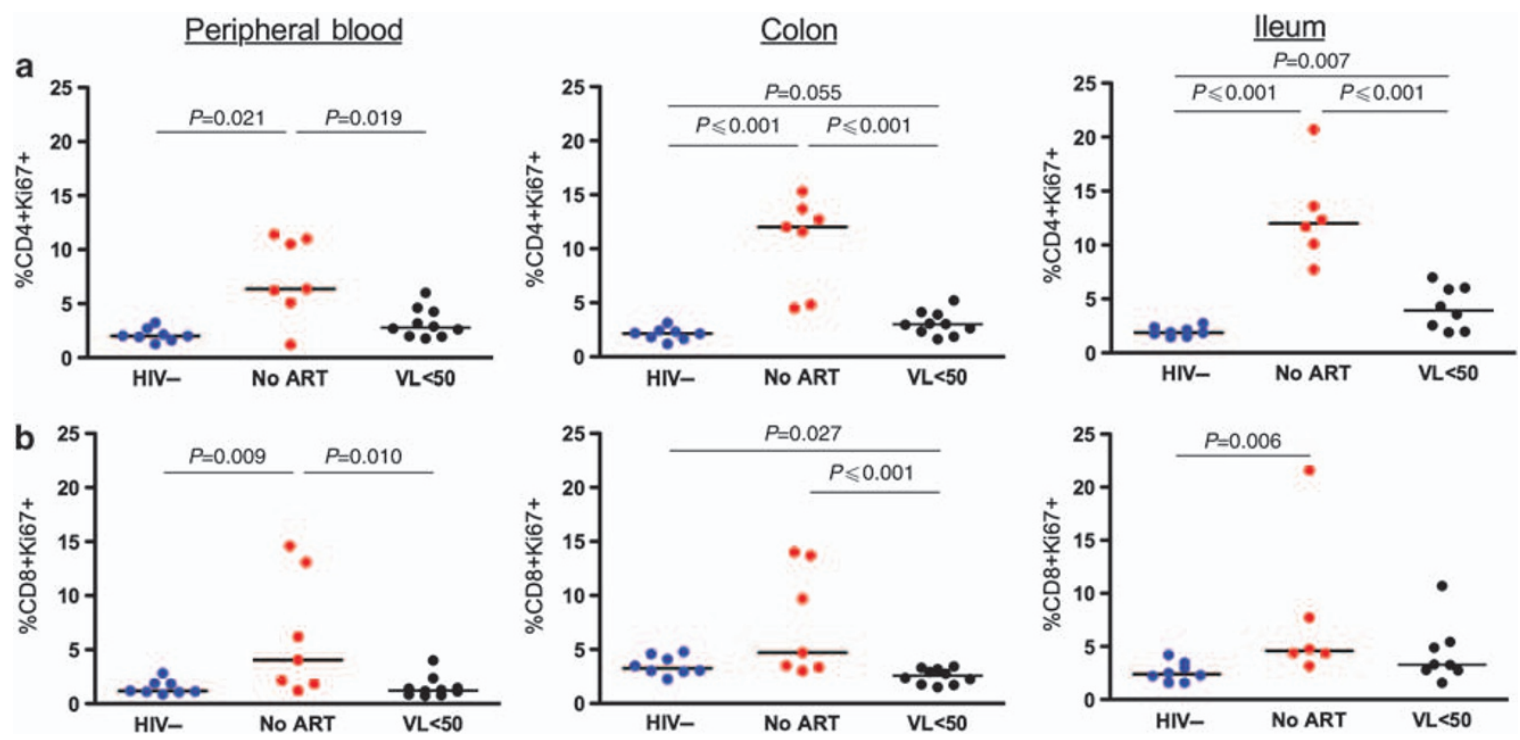

Figure 4 The percentage of cycling (a) CD4 + and (b) CD8 + T cells (Ki67+) in peripheral blood and gut compartments. Included in the analysis are participants who had Ki67 subset information for both PB and colon ( $8 \mathrm{HIV}-, 7 \mathrm{No}$ ART and $10 \mathrm{VL}<50$ ). Of these individuals, ileum measurements were not available for one No ART and two $\mathrm{VL}<50$ participants. Black bars indicate median values.

Table 2 Results of Spearman rank tests comparing the percentage of cycling CD4 + and CD8 + T cells in PB and GI sites with other cellular and viral measurements among HIV-infected individuals

\begin{tabular}{|c|c|c|c|c|c|c|}
\hline & $\% C D 4+$ PB & $\begin{array}{c}\text { CD4 + T cells per } \\
\mu l(\text { PB) }\end{array}$ & $\%$ CD4 + colon & $\begin{array}{c}\text { CD4 + T cells } \\
\text { per gm of tissue } \\
\text { (colon) }\end{array}$ & $\%$ CD4 + ileum & $\begin{array}{l}\text { CD4 T cells per } \\
\text { gm of tissue } \\
\text { (ileum) }\end{array}$ \\
\hline \multirow{2}{*}{$\begin{array}{l}\% \mathrm{CD} 4+\mathrm{Ki} 67+{ }^{\mathrm{a}} \\
\text { (at matching } \\
\text { site) }\end{array}$} & -0.59 & -0.73 & -0.62 & -0.43 & -0.51 & -0.39 \\
\hline & 0.004 & $<0.001$ & 0.009 & 0.114 & 0.061 & 0.184 \\
\hline $\begin{array}{l}\text { (at matching } \\
\text { site) }\end{array}$ & \multicolumn{3}{|c|}{ Plasma HIV-RNA (copies per ml) } & \multicolumn{3}{|c|}{ Colonic HIV-RNA (copies per mg) } \\
\hline $\begin{array}{l}\text { \%CD4 + Ki67 + } \\
\text { PB }\end{array}$ & \multicolumn{3}{|c|}{0.70} & \multicolumn{3}{|c|}{0.41} \\
\hline \multirow{2}{*}{$\begin{array}{l}\% \mathrm{CD} 8+\mathrm{Ki} 67+ \\
\mathrm{PB}\end{array}$} & \multicolumn{3}{|c|}{0.78} & \multicolumn{3}{|c|}{0.54} \\
\hline & \multicolumn{3}{|c|}{0.008} & \multicolumn{3}{|c|}{0.171} \\
\hline \multirow{2}{*}{$\begin{array}{l}\% \mathrm{CD} 8+\mathrm{Ki} 67+ \\
\text { colon }\end{array}$} & \multicolumn{3}{|c|}{0.07} & \multicolumn{3}{|c|}{0.49} \\
\hline & \multicolumn{3}{|c|}{0.880} & \multicolumn{3}{|c|}{0.356} \\
\hline
\end{tabular}

${ }^{a}$ Analysis of HIV + groups only.

Correlation analyses of plasma and tissue HIV-RNA only included No ART patients.

and TI; however, the relationship did not appear to be linear (Supplementary Figure 3).

Among viremic participants, there was a statistically significant correlation between both the percentage of $\mathrm{CD} 4+\mathrm{Ki} 67+$ and $\mathrm{CD} 8+\mathrm{Ki} 67+\mathrm{T}$ cells in $\mathrm{PB}$ and plasma
HIV-RNA. However, neither the percent of CD4 + Ki67 + nor of CD8 + Ki67 + in colon was associated with colonic or plasma HIV-RNA (Table 2). Both the percentages of $\mathrm{CD} 4+\mathrm{Ki} 67+$ and CD8 + Ki67 + in PB correlated with the same subset in colon $(r=0.69$ and $r=0.68 ; P \leqslant 0.001)$. 

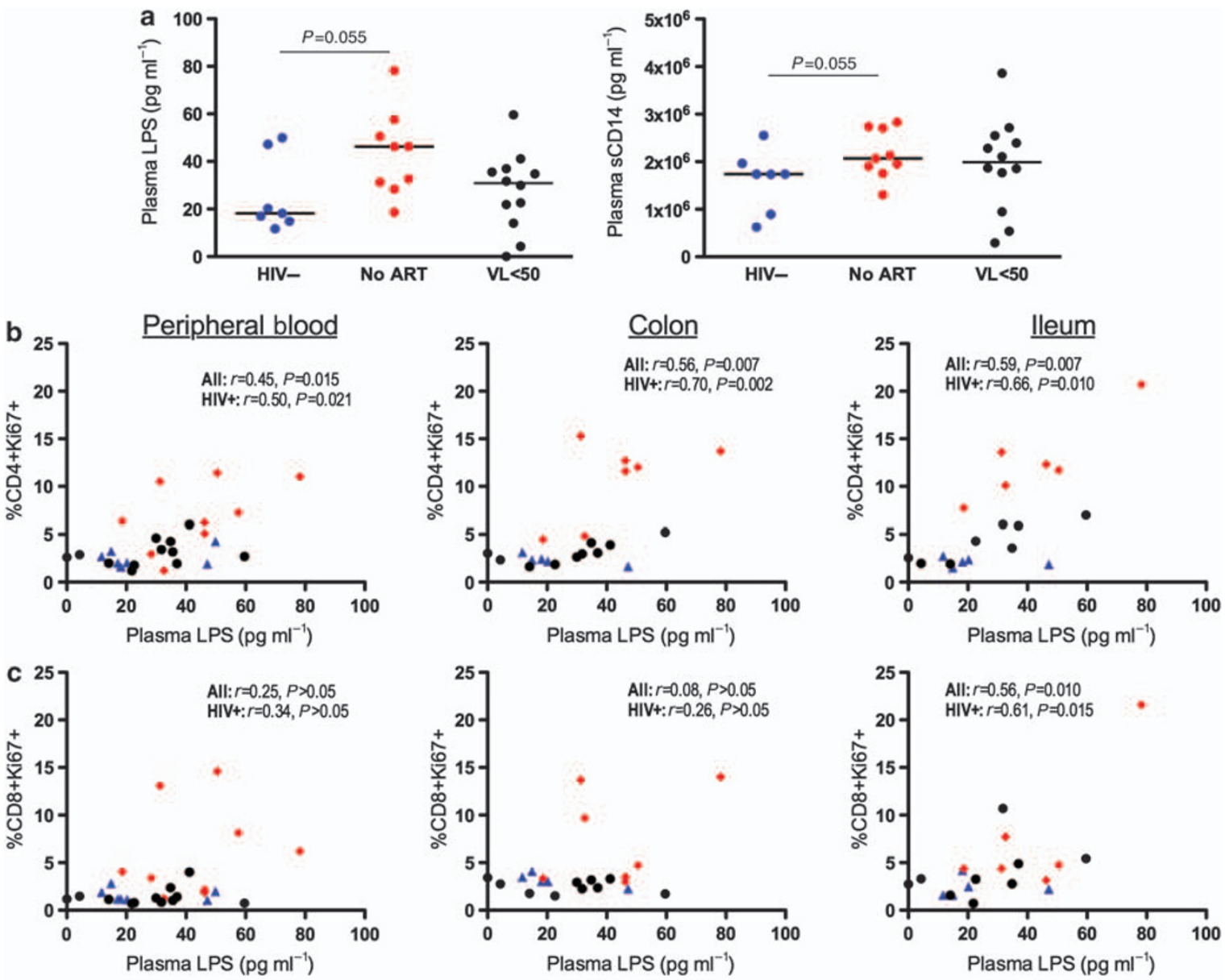

\section{$\triangle$ HIV- $\bullet$ No ART $\bullet \mathrm{VL}<50$}

Figure 5 (a) Plasma levels of LPS and SCD14 in 7 HIV- (blue triangles), 9 No ART (red diamonds), and 12 VL $<50$ (black circles) individuals. Black bars indicate median values. (b,c) Correlation between LPS levels and the percentage of CD4+Ki67+ and CD8+Ki67+ T cells in peripheral blood (left column), colon (middle column), and ileum (right column). Colon values were not available for an additional two participants from each group. Spearman rank tests were used to determine correlation and $P$-values.

Plasma and colonic HIV-RNA levels did not correlate $(r=0.39$, $P=0.230)$.

\section{The proportion of cycling CD4+, but not CD8+,T cells in both PB and colon was strongly associated with plasma LPS levels}

Plasma levels of LPS, a marker of microbial translocation, and soluble CD14 (sCD14), a marker of monocyte activation after stimulation by LPS, ${ }^{16}$ were quantified. There was no statistically significant difference in plasma or soluble CD14 levels between the three groups, although both markers were higher in viremic patients as compared with controls (LPS, No ART: $46.2 \mathrm{pg} \mathrm{ml}^{-1}$ vs. HIV-: $18.2 \mathrm{pg} \mathrm{m}^{-1}$; sCD14, No ART: $2.07 \times 10^{6} \mathrm{pg} \mathrm{ml}^{-1}$ vs. HIV-: $1.74 \times 10^{6} \mathrm{pg} \mathrm{ml}^{-1} ; P=0.055$ for both comparisons) (Figure 5a). Plasma LPS levels did correlate with the percent of cycling $(\mathrm{Ki} 67+) \mathrm{CD} 4+\mathrm{T}$ cells in $\mathrm{PB}$ and both gut sites (Figure $5 \mathbf{b}$ ), but not with the proportion of cycling CD8 + T cells in either $\mathrm{PB}$ or colon (Figure 5c). These observations were consistent whether the analysis included all three groups or was restricted to HIV-infected individuals. There was no association between
sCD14 and cycling CD4 + or CD8 + T cells in either PB or colon.

Multivariate linear regression analysis was performed to further delineate the relationship between LPS and CD4 + T-cell cycling in the gut. LPS remained statistically significantly associated with the proportion of CD4 $+\mathrm{Ki} 67+\mathrm{T}$ cells in the colon after controlling for either the proportion $(P=0.019)$ or absolute number $(P=0.002)$ of $\mathrm{CD} 4+\mathrm{T}$ cells at the same site.

\section{DISCUSSION}

In this study, we found that after prolonged ART, the cycling of $\mathrm{CD} 4+$ and $\mathrm{CD} 8+\mathrm{T}$-cell populations was decreased or normalized and the number of CD4 $+\mathrm{T}$ cells was restored in the gut mucosa of HIV-infected patients. The gut CD4 + T-cell immune restoration was also reflected in $\beta 7$ expression on peripheral blood CD4 + T cells. Cycling of gut CD4 + T cells was associated with plasma LPS levels but not with plasma or tissue HIV-RNA levels, indicating that gut T-cell activation in the gut may be interconnected with microbial translocation.

In concordance with previous studies, ${ }^{4,10,17,21}$ we observed that the percentage of CD $4+\mathrm{T}$ cells in both colon and ileum 
remained significantly lower than that of HIV-uninfected controls despite effective prolonged ART. However, the persistence of a high proportion of CD8 + T cells in HIV-infected patients appeared to result in an underestimation of CD4 + T-cell reconstitution. We attempted to address this issue by enumerating the absolute number of CD4 $+\mathrm{T}$ cells and observed no difference in absolute CD4 + T-cell numbers between HIV-uninfected controls and treated patients at both gut sites. Our findings are in agreement with recent studies using both immunohistochemistry and flow cytometric analyses; ${ }^{19,20,32}$ some of these have suggested that gut CD4 + T-cell reconstitution may even exceed what occurs in peripheral blood. ${ }^{20,32}$ We noted significant differences in the relative and absolute size of T-cell populations in colon and TI, but the proportions of both CD $4+$ and CD $8+\mathrm{T}$ cells at the two GI locations were strongly correlated across all study groups. This indicates that relative comparisons of CD4+ T-cell reconstitution between HIV-infected and HIV-uninfected individuals in colon and TI may lead to similar conclusions, but that direct comparisons of studies sampling different GI sites may not be feasible.

It has also been proposed that initiating ART therapy during acute infection may result in more rapid and complete reconstitution of the CD4 + T-cell population in the gut. ${ }^{21-23}$ Three of the four patients in this study who reconstituted their CD4+ $\mathrm{T}$-cell counts in the colon to values higher than the median of the HIV-uninfected group had peripheral nadir CD4 + T-cell counts of less than 250 cells per microliter. This suggests that $\mathrm{CD} 4+\mathrm{T}$-cell restoration may occur despite substantial disease progression before ART initiation.

The mechanism by which T-cell reconstitution in the gut mucosa occurs remains unclear. Microarray analysis of jejunal lymphocytes has shown that the expression of genes related to cell trafficking, but not local proliferation, were increased in patients receiving ART as compared with uninfected controls. ${ }^{10}$ Our finding that the proportion of cycling $\mathrm{T}$ cells is similar in treated patients and uninfected controls also suggests that $\mathrm{CD} 4+\mathrm{T}$-cell populations in the gut may be sustained by trafficking of $\mathrm{T}$ cells to the gut mucosa as opposed to local proliferation after prolonged therapy. However, there may be transient increases in T-cell cycling at earlier time points after ART initiation that may contribute to T-cell reconstitution, a possibility that could not be tested in this study because of our enrollment of chronically infected individuals treated with prolonged ART. Further longitudinal studies may thus be necessary to determine factors associated with substantial recovery of CD $4+\mathrm{T}$ cells in the gut mucosa and the clinical significance of this immune reconstitution.

This study is the first to examine the relationship between $\beta 7 \mathrm{hi}$ expression in the blood and gut $\mathrm{T}$-cell populations in humans and in the context of ART. Evidence from the rhesus macaque model of SIV infection has suggested that CD4 + T cells expressing the lymphocyte homing marker $\alpha 4 \beta 7$ may have a role in gut pathogenesis and immune reconstitution, and therefore may serve as a surrogate marker for intestinal CD $4+\mathrm{T}$ cells.${ }^{26}$ Our observation that a strong association exists between $\beta 7 \mathrm{hi}$, but not CCR5, expression on blood CD4 + T cells and CD4 + T-cell populations at both gut sites is consistent with this hypothesis. Interestingly, in this cross-sectional study we observed similar associations between both the proportion and absolute number of the CD4 + T-cell subset in blood and the same populations in colon and ileum, suggesting that measurement of the $\alpha 4 \beta 7$ subset may not be necessary to track intestinal CD4 + T-cell populations in the context of chronic untreated infection or after prolonged ART. However, given that during acute infection CD4 + T-cell depletion occurs more rapidly in the gut as compared with blood, ${ }^{4,6,8-14}$ it will be of great interest to evaluate the association between $\alpha 4 \beta 7$ expression in the peripheral blood and gut CD4 + T-cell populations during acute HIV infection.

Unlike in peripheral blood, where the proportion of cycling CD4 + and CD8 + T cells was associated with plasma HIVRNA, we observed no association between T-cell cycling in the colon and viral burden in either tissue or plasma among viremic, untreated patients. This suggests that the factors that most significantly impact T-cell proliferation may be different in the two compartments. In peripheral blood, increased T-cell cycling in untreated infection is thought to be the result of HIV-induced immune activation as well as a homeostatic response to $\mathrm{T}$-cell depletion. ${ }^{29}$ In our treated group, the median proportion of activated $\mathrm{CD} 4+$ and $\mathrm{CD} 8+\mathrm{T}$ cells (as determined by co-expression of HLA-DR and CD38) in blood was significantly lower than that of the untreated group (data not shown). Therefore, it is likely that the prolonged suppression of viral replication with therapy results in a decrease in peripheral immune activation and a subsequent reduction in T-cell cycling. In contrast, in the gut mucosa, it is possible that restoration of the $\mathrm{CD} 4+\mathrm{T}$-cell population itself may result in a decrease in $\mathrm{CD} 4+\mathrm{T}$-cell cycling by reducing homeostatic signals that trigger local proliferation. In our HIV-infected groups, the proportion of cycling CD4+ $\mathrm{T}$ cells in the colon correlated with the proportion of CD4+ gut $\mathrm{T}$ cells at the same site, a trend that has been observed in blood both in this study and others. ${ }^{28,33}$ However, it was not associated with the number of gut mucosal CD $4+\mathrm{T}$ cells, and all of our treated patients had a relatively small proportion of cycling CD $4+\mathrm{T}$ cells despite showing a wide range of absolute $\mathrm{CD} 4+\mathrm{T}$-cell counts, arguing against proliferation as a homeostatic response to T-cell depletion. Therefore, it is possible that the suppression of viral replication and, in turn, local immune activation (or vice versa), in addition to other unknown mechanisms, may also be contributing to the decrease in T-cell cycling observed in the colon after prolonged therapy.

To our knowledge, this is the first analysis of possible associations between plasma levels of LPS and markers of T-cell cycling and activation in the gut mucosa. Previous research has examined the relationship between LPS levels and peripheral markers of activation and proliferation. A significant correlation between Ki67 + CD4 + and CD8 + T cells in peripheral blood and plasma LPS levels was observed among treated patients and specifically immunological non-responders. ${ }^{34}$ Although this study is limited by its correlative nature, we found that, among all three groups in our study, the proportion of cycling CD4 + but not CD8 + T cells in colon was associated with plasma levels of LPS, a marker 
of microbial translocation, suggesting an association between LPS and cellular immune activation in the CD4 + T-cell subset both systemically and locally in the gut mucosa. It is unclear whether LPS is a cause $\mathrm{e}^{16}$ or an effect ${ }^{35,36}$ of immune activation. If LPS were the cause of activation-induced cell death through stimulation of the innate immune system, it would be expected to induce increased cycling of both CD4 + and CD8 + T cells. It is possible that microbial translocation may instead be a consequence of HIV-mediated damage to the gut mucosal barrier because of increased local immune activation and CD4 + T-cell turnover. In a multivariate analysis, plasma LPS remained significantly associated with colon CD4 + T-cell cycling even after controlling for either colon CD4 + T-cell proportion or absolute number. Measurements of gut tissue LPS, which has been shown in SIV-infected macaques using immunohistochemistry (Jacob D Estes, personal communication), would be important for determining the biological mechanism of the interaction between microbial translocation and T-cell cycling as well as the clinical significance of this relationship in the context of HIV infection.

In conclusion, we observed that substantial restoration of the gut CD4 + T-cell population can occur after therapy and is largely reflected in $\beta 7$ expression on blood CD $4+\mathrm{T}$ cells. Our findings also suggest that $\mathrm{T}$-cell cycling decreases significantly in the gut mucosa in response to prolonged ART and may be regulated by both homeostatic and local stimuli such as those resulting from microbial products.

\section{METHODS}

\section{Subjects}

Biopsies from colon and terminal ileum as well as peripheral blood samples were collected from 14 viremic HIV-infected individuals (No ART), $12 \mathrm{HIV}$-infected patients treated with prolonged ART (longer than 5 years) $(\mathrm{VL}<50)$, and $10 \mathrm{HIV}$-uninfected controls (HIV-). All participants provided informed consent under an IRB-approved NIH Protocol (registered at www.clinicaltrials.gov, NCT no. 00001471). Plasma viral loads were determined by ultrasensitive bDNA assay (Versant HIV-1 version 3.0, Siemens, New York City, NY).

\section{Biopsy processing}

To collect colonic and terminal ileum tissue, subjects underwent a routine endoscopy procedure under moderate conscious sedation. Approximately 30 endoscopic biopsies were randomly taken from the gut mucosa at each location with 16-20 processed for flow cytometric analysis. In groups of five, the biopsies were weighed, placed in $500 \mu \mathrm{l}$ of medium containing RPMI (Mediatech, Herndon, VA) with $10 \%$ heat-inactivated fetal bovine serum. Samples were then digested using $1 \mathrm{mg} \mathrm{ml}^{-1}$ collagenase (Sigma-Aldrich, St Louis, MO) and 2,000 U DNAse I (Invitrogen, Carlsbad, CA) for $30 \mathrm{~min}$ at $37^{\circ} \mathrm{C}$ or $250 \mathrm{U}$ benzoase (Novagen, Madison, WI) for $40 \mathrm{~min}$ at $37^{\circ} \mathrm{C}$ before being filtered through a $40 \mu \mathrm{m}$ screen. After being washed twice with the $10 \%$ FBS medium, the resulting cell suspension was counted using a Beckman Coulter Counter to obtain the number of total viable cells. Histopathological evaluation confirmed sampling of isolated lymphoid follicles in the colon and Peyer's patches/gut-associated lymphoid tissue in the ileum, although the biopsies primarily consisted of lamina propria lymphocytes (data not shown).

\section{HIV-1 Viral RNA extraction from gut tissue}

Two biopsies from colon and terminal ileum were snap frozen on dry ice for measurement of tissue HIV-RNA. The samples were weighed and then homogenized in AVL buffer (QIAamp Viral mini kit cat no. $52,904)$ using a mini mortar and pestle. Extraction was completed per kit instructions. The Siemens Quantiplex HIV-1 3.0 assay was used to measure HIV-1 RNA copy number. Results are expressed as copies per milligram of tissue.

\section{Immunophenotyping}

Immunophenotypical analysis was performed on whole blood, cryopreserved peripheral blood mononuclear cells (in the case of the $\beta 7$ and CCR 5 staining only), and cells extracted from the gut biopsies as described elsewhere. ${ }^{37}$ Comparison of $\beta 7$ and CCR5 staining between fresh peripheral blood mononuclear cells, cryopreserved PBMC stained immediately after thawing, and cryopreserved peripheral blood mononuclear cells rested for $3 \mathrm{~h}$ before staining showed that a 3-hour rest maintained $\beta 7$ and CCR5 expression that is often downregulated with freezing (Supplementary Figure 4). The following antibodies were used for immunophenotyping of whole blood samples: anti-CD3 FITC (clone SK7), anti-CD4 PerCP or APC (clone SK3) and anti-CD8 PerCP (clone SK1), (BD Biosciences, San Jose, CA), and anti-Ki67 PE (clone B56) (BD Pharmingen, San Jose, CA). The following additional antibodies were used for staining of frozen peripheral blood mononuclear cells (PMBC): anti-CD3 APC-Cy7 (clone SK7), anti- $\beta 7$ integrin PE-Cy5 (clone FIB504), anti-CCR5 PE (clone 2D7), CD27 FITC (clone M-T271) (BD Pharmingen), CD45RO APC (clone UCHL1) (BD Biosciences) and anti-CD4 Qdot 605 (clone S3.5) (Invitrogen). Samples were acquired using a FACSCalibur or an LSRII flow cytometer (BD Pharmingen). The data were analyzed using FlowJo software version 8 (Tree Star, San Carlos, CA).

\section{Calculation of absolute numbers of gut T-cell subsets}

Absolute numbers of CD4 + and CD8 + T-cell subsets per gram of gut tissue were calculated by multiplying the total viable cell count by percentages obtained from flow cytometric analysis. The total cell count per gram of tissue was calculated by dividing the viable cell count as determined by Coulter Counter (Beckman Coulter, Fullerton, CA) by the tissue weight. This proportion was then multiplied by the percent of cells in the live lymphocytes gate and that number was subsequently multiplied by the percent of CD3 + lymphocytes. The absolute number of gut CD3 + T cells was then used in conjunction with the subset percentages to determine the absolute number of each T-cell subset per gram of biopsy tissue.

\section{Measurement of lipopolysaccharide and soluble CD14 (sCD14) levels}

LPS levels were quantified in duplicate by dilution of plasma samples collected in EDTA tubes to 10\% with endotoxin-free water and subsequent heat inactivation of plasma proteins for $15 \mathrm{~min}$ at $80^{\circ} \mathrm{C}$ using a Limulus Amebocyte Assay (Lonza Group, Switzerland). Background was then subtracted and LPS levels were calculated by first setting the $y$-intercept for the standard regression line at zero and then by the manufacturer's recommended protocol. Soluble CD14 (sCD14) plasma levels were measured in duplicate using a commercially available ELISA assay (R\&D Systems, Minneapolis, MN), and analyzed according to the manufacturer's recommended procedure.

\section{Statistical analyses}

Values are expressed as medians with interquartile ranges. Kruskal-Wallis and Wilcoxon matched pairs tests were used for between and within group comparisons, respectively. Spearman's Rank tests were used to calculate correlation coefficients. Owing to the exploratory nature of the work, all $P$-values of 0.05 are shown; however, to account for multiple comparisons, $P<0.01$ was considered statistically significant by the authors. Analyses were performed using Prism v5.0 (GraphPad Software, La Jolla, CA).

SUPPLEMENTARY MATERIAL is linked to the online version of the paper at http://www.nature.com/mi 


\section{ACKNOWLEDGMENTS}

We acknowledge Andrew Redd, Vitaly Ganusov and Michele Di Mascio for their helpful comments and discussion, Jason Brenchley and Danny Douek for their advice regarding the LPS assay, and the staff of the OP8 clinic and the Gl suite at the NIH Clinical Center for their assistance with patient recruitment and care. Special thanks to all study participants who volunteered for research gut biopsy procedures. This research was supported in part by the Intramural Program of the NIH, NIAID and Critical Care Medicine Department. In addition, this project has been funded in whole or in part with federal funds from the National Cancer Institute, National Institutes of Health, under Contract No. HHSN261200800001E. The content of this publication does not necessarily reflect the views or policies of the Department of Health and Human Services, nor does it mention of trade names, commercial products, or organizations imply endorsement by the US Government.

\section{DISCLOSURE}

The authors declared no conflict of interest.

(C) 2010 Society for Mucosal Immunology

\section{REFERENCES}

1. Lapenta, C. et al. Human intestinal lamina propria lymphocytes are naturally permissive to HIV-1 infection. Eur. J. Immunol. 29, 1202-1208 (1999).

2. Anton, P.A. et al. Enhanced levels of functional HIV-1 co-receptors on human mucosal T cells demonstrated using intestinal biopsy tissue. AIDS. 14, 1761-1765 (2000).

3. Poles, M.A., Elliott, J., Taing, P., Anton, P.A. \& Chen, I.S. A preponderance of CCR5(+) CXCR4(+) mononuclear cells enhances gastrointestinal mucosal susceptibility to human immunodeficiency virus type 1 infection. J. Virol. 75, 8390-8399 (2001).

4. Mehandru, S. et al. Primary HIV-1 infection is associated with preferential depletion of CD4+ T lymphocytes from effector sites in the gastrointestinal tract. J. Exp. Med. 200, 761-770 (2004).

5. Schnittman, S.M., Lane, H.C., Greenhouse, J., Justement, J.S., Baseler, M. \& Fauci, A.S. Preferential infection of CD4+ memory T cells by human immunodeficiency virus type 1: evidence for a role in the selective T-cell functional defects observed in infected individuals. Proc. Natl. Acad. Sci. USA. 87, 6058-6062 (1990).

6. Veazey, R.S. et al. Gastrointestinal tract as a major site of CD4+ T-cell depletion and viral replication in SIV infection. Science. 280, 427-431 (1998).

7. van Noesel, C.J., Gruters, R.A., Terpstra, F.G., Schellekens, P.T., van Lier, R.A. \& Miedema, F. Functional and phenotypic evidence for a selective loss of memory $T$ cells in asymptomatic human immunodeficiency virusinfected men. J. Clin. Invest. 86, 293-299 (1990).

8. Li, Q. et al. Peak SIV replication in resting memory CD4+ T cells depletes gut lamina propria CD4+ T cells. Nature. 434, 1148-1152 (2005).

9. Brenchley, J.M. et al. CD4+ T-cell depletion during all stages of HIV disease occurs predominantly in the gastrointestinal tract. J. Exp. Med. 200, 749-759 (2004).

10. Guadalupe, M. et al. Severe CD4+ T-cell depletion in gut lymphoid tissue during primary human immunodeficiency virus type 1 infection and substantial delay in restoration following highly active antiretroviral therapy J. Virol. 77, 11708-11717 (2003).

11. Lim, S.G., Condez, A., Lee, C.A., Johnson, M.A., Elia, C. \& Poulter, L.W. Loss of mucosal CD4 lymphocytes is an early feature of HIV infection. Clin. Exp. Immunol. 92, 448-454 (1993).

12. Mattapallil, J.J., Douek, D.C., Hill, B., Nishimura, Y., Martin, M. \& Roederer, M. Massive infection and loss of memory CD4+ T cells in multiple tissues during acute SIV infection. Nature. 434, 1093-1097 (2005).

13. Schneider, T., Jahn, H.U., Schmidt, W., Riecken, E.O., Zeitz, M. \& Ullrich, R. Loss of CD4 T lymphocytes in patients infected with human immunodeficiency virus type 1 is more pronounced in the duodenal mucosa than in the peripheral blood. Berlin Diarrhea/Wasting Syndrome Study Group. Gut. 37, 524-529 (1995).

14. Clayton, F., Snow, G., Reka, S. \& Kotler, D.P. Selective depletion of rectal lamina propria rather than lymphoid aggregate CD4 lymphocytes in HIV infection. Clin. Exp. Immunol. 107, 288-292 (1997).
15. Li, Q. et al. Simian immunodeficiency virus-induced intestinal cell apoptosis is the underlying mechanism of the regenerative enteropathy of early infection. J. Infect. Dis. 197, 420-429 (2008).

16. Brenchley, J.M. et al. Microbial translocation is a cause of systemic immune activation in chronic HIV infection. Nat. Med. 12, 1365-1371 (2006).

17. Mehandru, S. et al. Lack of Mucosal Immune Reconstitution during Prolonged Treatment of Acute and Early HIV-1 Infection. PLoS. Med. 3, e484 (2006).

18. Chun, T.W. et al. Persistence of HIV in gut-associated lymphoid tissue despite long-term antiretroviral therapy. J. Infect. Dis. 197, 714-720 (2008).

19. Macal, M. et al. Effective CD4+ T-cell restoration in gut-associated lymphoid tissue of HIV-infected patients is associated with enhanced Th17 cells and polyfunctional HIV-specific T-cell responses. Mucosal. Immunol. 1, 475-488 (2008).

20. Sheth, P.M. et al. Immune reconstitution in the sigmoid colon after longterm HIV therapy. Mucosal. Immunol. 1, 382-388 (2008).

21. Guadalupe, M. et al. Viral suppression and immune restoration in the gastrointestinal mucosa of human immunodeficiency virus type 1 -infected patients initiating therapy during primary or chronic infection. J. Virol. 80, 8236-8247 (2006).

22. George, M.D., Reay, E., Sankaran, S. \& Dandekar, S. Early antiretroviral therapy for simian immunodeficiency virus infection leads to mucosal $\mathrm{CD} 4+\mathrm{T}$-cell restoration and enhanced gene expression regulating mucosal repair and regeneration. J. Virol. 79, 2709-2719 (2005).

23. Pandrea, I.V. et al. Acute loss of intestinal CD4+ T cells is not predictive of simian immunodeficiency virus virulence. J. Immunol. 179, 3035-3046 (2007).

24. Pakker, N.G. et al. Biphasic kinetics of peripheral blood T cells after triple combination therapy in HIV-1 infection: a composite of redistribution and proliferation. Nat. Med. 4, 208-214 (1998).

25. Sousa, A.E., Chaves, A.F., Doroana, M., Antunes, F. \& Victorino, R.M. Kinetics of the changes of lymphocyte subsets defined by cytokine production at single cell level during highly active antiretroviral therapy for HIV-1 infection. J. Immunol. 162, 3718-3726 (1999).

26. Wang, X., Xu, H., Gill, A.F., Pahar, B., Kempf, D., Rasmussen, T., Lackner, A.A. \& Veazey, R.S. Mucosal. Immunol. 2, 518-526 (2009).

27. Tenner-Racz, K. et al. The unenlarged lymph nodes of HIV-1-infected, asymptomatic patients with high CD4 T-cell counts are sites for virus replication and CD4 T-cell proliferation. The impact of highly active antiretroviral therapy. J. Exp. Med. 187, 949-959 (1998).

28. Sachsenberg, N. et al. Turnover of CD4+ and CD8+ T lymphocytes in HIV1 infection as measured by Ki-67 antigen. J. Exp. Med. 187, 1295-1303 (1998).

29. Hazenberg, M.D. et al. T-cell division in human immunodeficiency virus $(\mathrm{HIV})-1$ infection is mainly due to immune activation: a longitudinal analysis in patients before and during highly active antiretroviral therapy (HAART). Blood. 95, 249-255 (2000).

30. Arthos, J. et al. HIV-1 envelope protein binds to and signals through integrin á4â7, the gut mucosal homing receptor for peripheral T cells. Nat. Immunol. 9, 301-309 (2008).

31. Salmi, M. \& Jalkanen, S. Molecules controlling lymphocyte migration to the gut. Gut. 45, 148-153 (1999).

32. Connick, E. et al. Substantial CD4+ T-cell recovery and reconstitution of tissue architecture in gut associated lymphoid tissue (GALT) in advanced HIV1 infection following initiation of HAART [abstract]. Conference on Retroviruses and Opportunistic Infections; 26 February (2007), Los Angeles, CA

33. Anthony, K.B. et al. Incomplete CD4 T-cell recovery in HIV-1 infection after 12 months of highly active antiretroviral therapy is associated with ongoing increased CD4 T-cell activation and turnover. J. Acquir. Immune. Defic. Syndr. 33, 125-133 (2003).

34. Marchetti, G. et al. Microbial translocation is associated with sustained failure in CD4+ T-cell reconstitution in HIV-infected patients on long-term highly active antiretroviral therapy. AIDS. 22, 2035-2038 (2008).

35. Redd, A.D. et al. Microbial translocation, the innate cytokine response, and HIV-1 disease progression in Africa. Proc. Natl. Acad. Sci. USA. 106, 6718-6723 (2009)

36. Paiardini, M., Frank, I., Pandrea, I., Apetrei, C. \& Silvestri, G. Mucosal immune dysfunction in AIDS pathogenesis. AIDS Rev. 10, 36-46 (2008).

37. Sereti, I. et al. CD4 T-cell expansions are associated with increased apoptosis rates of T lymphocytes during IL-2 cycles in HIV infected patients. Aids. 15, 1765-1775 (2001). 